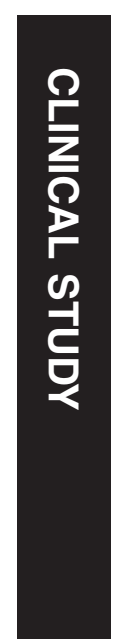

\title{
Clinical features and long-term progression of reticular pseudodrusen in age- related macular degeneration: findings from a multicenter cohort
}

'Department of Ophthalmology-Centro Hospitalar e Universitário de Coimbra, Coimbra, Portugal

${ }^{2}$ Association for Innovation and Biomedical Research on Light and Image

(AIBILI), Coimbra, Portugal

${ }^{3}$ Center for Experimental Medicine, Queen's University Belfast, Belfast, UK

${ }^{4}$ Department of Clinical Science 'Luigi Sacco', Eye Clinic, Sacco Hospital, University of Milan, Milan, Italy

${ }^{5}$ Faculty of Medicine, University of Coimbra, Coimbra, Portugal

Correspondence: Dr JQ Gil, Department of Ophthalmology-Centro Hospitalar e Universitário de Coimbra, Praceta Prof.

Mota Pinto, Coimbra 3000075, Portugal

Tel: +00 351239701182 Fax: +00 35123982666.

E-mail: joaomqgil@

hotmail.com

Received: 17 January 2016 Accepted in revised form: 2 August 2016

Published online:

21 October 2016

\begin{abstract}
Purpose To determine whether reticular pseudodrusen (RPD) confer a long-term increased risk of progression to late agerelated macular degeneration (AMD) in the fellow eye of patients with unilateral wetAMD.

Patients and methods This was a multicenter, combined prospective and retrospective, longitudinal, observational, study. Patients with wet-AMD in one eye were recruited from two centers and evaluated on the risk of progression to lateAMD in the second eye (study eye). A minimum follow-up of 5 years was required, unless progression occurred first. Baseline retinal profile of patients was evaluated using multimodal imaging. Baseline images were graded by two separate centers.

Results We recruited 88 patients (48 female) with a mean age of $75.6 \pm 7.1$ years and mean follow-up of $65.7 \pm 20.9$ months. Baseline prevalence of RPD was $58 \%(n=51)$. There was no statistically significant association of RPD with increased age $(P=0.29)$ or sex distribution $(P=0.39)$. The most sensitive image modality for RPD was IR $(\mathbf{9 3} \%)$, followed by FAF (92\%), OCT (74\%, RF (33\%) and CFP (29\%). After 5 years, $54.50 \%(n=48)$ of the study eyes progressed to late-AMD. Of those, $81.25 \%(n=39)$ developed CNV and $18.75 \%(n=9)$ geographic atrophy. After correcting for age and sex, the presence of RPD was significantly associated with development of late-stage AMD (OR $=2.55$, $P=0.03$ ).

Conclusion A multimodal approach is mandatory for RPD detection. RPD are highly
\end{abstract}

JQ Gil'1,2, JP Marques ${ }^{1,2}$, R Hogg $^{3}$, C Rosina $^{4}$, ML Cachulo ${ }^{1,2,5}$, A Santos ${ }^{1,2}$, G Staurenghi ${ }^{4}$, U Chakravarthy ${ }^{3}$ and R Silva ${ }^{1,2,5}$ prevalent in the fellow eyes of patients with unilateral neovascular AMD. Presence of RPD is associated with increased long-term risk of progression, highlighting the importance of comprehensive multimodal retinal imaging and careful monitoring of at-risk patients.

Eye (2017) 31, 364-371; doi:10.1038/eye.2016.207;

published online 21 October 2016

\section{Introduction}

Age-related macular degeneration (AMD) is one of the leading causes of blindness worldwide, particularly in industrialized countries, where over $35 \%$ of people over 80 years are thought to have intermediate or advanced AMD. ${ }^{1}$ Early stages of AMD are sometimes asymptomatic or very mildly symptomatic and have been characterized by the presence of drusen and pigmentary abnormalities. Classification of these features, based on color fundus photographs, has enabled us to stratify the risk of progression to the vision-threatening form of late-AMD. ${ }^{2}$

Reticular drusen were first described by Mimoun and Soubrane ${ }^{3}$ in 1990 as a yellowish interlacing network that was best seen with blue-light fundus photography. They were initially thought to originate from below the RPE, like typical drusen, and were included in the Wisconsin Age-Related Maculopathy Grading System as a specific form of drusen: 'reticular drusen'. ${ }^{4}$ With the advent of spectraldomain OCT, it was possible to locate RPD, not under, but above the RPE line. ${ }^{5}$ This meant that, despite similar appearance, pseudodrusen were in reality clearly different from soft drusen. 
Since its initial description, there has also been great interest in further characterization of RPD imaging. RPD are difficult to identify in clinical fundoscopic examination and poorly visualized with color fundus photographs. ${ }^{6}$ Introduction of infra-red (IR) reflectance scans, fundus autofluorescence (FAF), and red-free imaging (RF) has helped to better characterize these lesions. ${ }^{7}$ Difficulties in imaging acquisition and recognition are probably responsible for the remarkable variation in reported prevalence.

As a whole, we believe that RPD presence remains an underreported and underresearched retinal phenotype. The expansion of imaging techniques available is only now beginning to unveil the real nature of these lesions and driving a renewed interest in studying its impact on the clinical course of AMD patients. An example of this are the papers recently published by Finger et $a l^{8}$ and Hogg et $a l^{9}$ which reported on the risk to progression conferred by RPD to fellow eyes in patients diagnosed with unilateral choroidal neovascularization (CNV). An increased risk was found on eyes presenting with RPD, despite limited follow-up available. Given the importance of establishing long-term prognosis for patients with early-AMD, we wanted to further expand our knowledge on the significance of RPD by observing a longer followup in this specific high-risk group.

The purpose of this study is to assess the impact of RPD as an individual lesion on the 5-year risk of progression to late-stage AMD.

\section{Materials and methods}

\section{Population}

This was a multicenter, combined prospective and retrospective, longitudinal, observational study. Patients were recruited from two centers (Coimbra and Milan) as part of two prospective, observational, non-randomized, non-interventional, multicenter studies: the CNV Markers study (NCT00801541) and the Early Markers study (NCT00902785). An educational grant was provided by Pfizer Inc. (Pfizer, Brooklyn, NY, USA) to fund the two studies. In both studies consecutive male and female patients over 50 years of age (at the time of baseline observation) with a diagnosis of neovascular AMD in one eye (non-study eye) and no signs of late-AMD in the fellow eye (study eye) were studied with the aim of identifying retinal markers of progression to late-AMD. Eligibility criteria were similar for both studies and were as follows: CNV or macular scar due to AMD in the nonstudy eye; study eye with no CNV or geographic atrophy (GA); best corrected visual acuity (BCVA) greater than 20/40 or better than 70 ETDRS letters ('early markers' study only); sufficiently clear ocular media and adequate pupillary dilatation to permit good-quality fundus imaging of the study eye. All patients had to be willing and able to comply with scheduled visits, laboratory tests, and other trial procedures. Exclusion criteria for recruitment included any of the following: evidence of $\mathrm{CNV}$ in the study eye, significant media opacities (evidence of cataract likely to need surgery within the following 2 years was also an exclusion criteria), history of other ocular disease (including a refraction higher than -8 diopter power), medical conditions that would interfere with the patient's ability to complete the trial, any treatment with an ocular or systemic investigational agent in the past 60 days for any medical condition ('early markers' study only) or any intraocular surgery in the study eye within 60 days prior enrollment ('CNV markers' study only). Fluorescein angiography (FA) was used as the gold standard for detection of conversion to neovascular AMD.

During the first 2 years assessment was made every 6 months after the baseline visit. After the first 2 years, patients were seen on a yearly basis unless symptoms were noted, in which case unscheduled visits were arranged.

All patients had signed an informed consent, in accordance with the Helsinki Declaration and after institutional review board approval, where they agreed that their clinical data might be assessed for scientific purposes.

Sample size was calculated based on previous data published by our group that considered patients with a minimum follow-up of 12 months. ${ }^{9}$ Calculation was made using a desired statistical power of the trial of 0.8 and a desired significance of 0.05 .

A retrospective research was conducted to identify patients with a minimum follow-up of 5 years, unless progression to late-stage AMD in the study eye had occurred first. Their clinical records were reviewed to collect clinical data and baseline visit images were assessed for the presence of RPD.

\section{Image acquisition}

The prevalence of RPD at the moment of baseline observation was assessed using a multimodal imaging approach. Modalities used included color fundus photography (CFP), RF, FAF, IR, and optical coherence tomography (OCT). All patients also performed indocyanine-green angiography (ICG) and FA for the detection of neovascular AMD. All the patients performed the three first imaging (CFP, RF, FAF) exams and roughly half of the participants performed all 5 image modalities, including IR and OCT. 
Color fundus photograph Digital CFP was captured using either a Zeiss FF450 (Zeiss, Oberkochen, Germany) fundus camera with a Nikon (Nikon, Tokyo, Japan) highresolution camera to produce a $30^{\circ}$ field of view, centered both on the fovea and optic disk, or a Topcon $50 \times$ fundus camera (Topcon, Tokyo, Japan).

FA and ICG Simultaneous FA and ICG were acquired using the HRA II (Heidelberg Retina Angiograph; Heidelberg Engineering, Heidelberg, Germany) with the $30^{\circ}$ field of view centered on the macula.

Fundus autofluorescence FAF imaging was performed with the same retina angiograph scanning laser ophthalmoscope following a standard protocol. For the acquisition of the FAF film, the ametropic corrector of the angiograph was first adjusted to focus on the macula and sensitivity was altered until clear images of the macula and optic nerve were obtained. Multiple single $30^{\circ}$ autofluorescence images were acquired, aligned, and averaged.

IR and OCT IR and OCT images were obtained with the Spectralis HRA (Heidelberg Engineering) + device.

\section{Definitions for multimodal image analysis of reticular pseudodrusen}

Recognition of the presence or absence of RPD was made based on previously reported features. In CFP, reticular drusen were defined as confluent drusen forming an interlacing ribbon-like network, with individual lesions usually $>125 \mu \mathrm{m}$ in diameter. ${ }^{10}$ On IR imaging, RPD were considered as groups of hyporeflectant lesions against a mildly hyperreflectant background, in welldefined and regular patterns. ${ }^{11,12}$ Reticular autofluorescence was defined as a group of ill-defined, hypo-fluorescent lesions against a background of mildly elevated AF. ${ }^{13}$ In RF, a reticular pattern was defined as light, interlacing network of lesions that were 125-250 nm wide. ${ }^{12}$ On OCT, RPD were identified as accumulation of hyper-reflective material in the outer retinal layer anterior to the RPE. ${ }^{7}$

\section{Image grading}

For each included patient, the study eye and the central macular field (field 2) were analyzed. To assess for RPD presence, CFP, RF, FAF, IR, and OCT images were evaluated. Presence of RPD was considered when visible in one or more image modes. Grading was performed using a new semi-automatic softwareRetmarker AMD—which was developed to assist the manual grading of different types of AMD lesions and to automatically compute their number, size, and location. Software-assisted manual grading was performed as previously described ${ }^{14,15}$ The system does not automatically detects lesions but provides the grader with several available tools in the grading process, namely drawing free forms of all lesion types, drawing pre-set circular marks of different sizes $(63 \mu \mathrm{m}, 125 \mu \mathrm{m}, 175 \mu \mathrm{m}, 250 \mu \mathrm{m}$, or $500 \mu \mathrm{m})$, displaying higher/lower degrees of magnification, measuring distances, controlling brightness, and contrast or superimposing different image modes for better identification of lesions. Quantification of lesions is displayed on screen in real time and then provided to the grader at the end of the grading process. In addition to a categorical depiction of variables, such as the number $(0,1-9,0-19$, and $\geq 20)$ and area occupied by drusen $(<1 \%,<10 \%,<25 \%,<50 \%$, and $\geq 50 \%)$, this software allows their quantification as continuous variables, thus reflecting the real (absolute) number and area of drusen. ${ }^{14}$

The International Classification Grading System ${ }^{16}$ was assumed for this software and five circles (C0, C1, C2, C:3, and $\mathrm{C} 4$ ) of $63,125,175,250$, and $500 \mu \mathrm{m}$ were available for lesions' grading. Before any measurement, each image was manually calibrated by the user using the optic disc diameter $(1.5 \mathrm{~mm})$ as a reference. Then, all AMD signs and RPD within a fixed area around the fovea (diameter, $6000 \mu \mathrm{m}$ ) were recorded. This area was delineated by a grid, consisting of three concentric circles and a right angled cross at $45^{\circ}$ and $135^{\circ}$ to the horizontal, which is adjusted according to the previous calibration. The diameters of the central, inner, and outer circle are $1000 \mu \mathrm{m}, 3000 \mu \mathrm{m}$, and $6000 \mu \mathrm{m}$, respectively, the same used by the International Classification System. ${ }^{16}$ Whenever RPD were visible but located outside the grid, they were manually graded and included so as to not miss any lesion.

Images from both centers were divided and graded independently. Images from 41 patients were graded by the Coimbra group, whereas images from 47 patients were graded by a third independent group from Belfast. All cases including IR and OCT images were graded in the Belfast center. In both centers, each class of images was assessed by two independent graders, beforehand trained and certified for AMD grading. Between-grader agreement was evaluated using the percentage of agreement (ie, number of images with the same classification) and the weighted $\kappa$-coefficient. The Landis and Koch criteria were used for the $\kappa$-values. Difficult cases, where the presence of RPD was questionable or where discrepancies existed, were re-graded in a side-byside manner. When discrepancies remained between the original and subsequent side-by-side grading, the images 
were adjudicated and verified by a senior specialist with grading experience.

\section{Statistical analysis}

Statistical analysis was performed using SPSS 21 software (SPSS Inc, Chicago, IL, USA). Baseline values are presented as mean $\pm \mathrm{SD}$ for continuous variables and as absolute value and percentage for discrete variables. Differences in baseline characteristics of patients with and without RPD were analyzed using independent samples Kruskis-Wallis test for continuous variables and $\chi^{2}$-test for bivariate variables. Associations between RPD and AMD progression were made using logistic regression techniques, after sex and age adjustments.

\section{Results}

A total of 88 participants (48 female) with unilateral CNV and no signs of late-AMD in the fellow eye were included. All patients were Caucasian and presented a mean age of $75.57 \pm 7.05$ years (52-93). Mean available follow-up was $65.65 \pm 20.90$ (14-94) months. Baseline mean BCVA in the study eye (non-affected eye) was $0.13 \pm 0.14 \log \mathrm{MAR}$, ranging from 0.48 to $0.00 \log \mathrm{MAR}$. Baseline characterization of the patients is summarized in Table 1.

\section{Multimodal reticular pseudodrusen assessment}

All patients had performed CFP, RF, and FAF but only $53.41 \%(n=47)$ had IR and OCT images available. When all cases were considered, the prevalence of RPD was 58\% $(n=51)$. The sub-group analysis of subjects with IR and OCT available revealed similar prevalence results (59.6\%, $n=28)$.

A comparison of the baseline characterization of patients with RPD $(n=51)$ and without RPD $(n=37)$ is summarized in Table 1 . No differences were noted in sex

Table 1 Baseline characteristics of patients with and without reticular pseudodrusen

\begin{tabular}{lrrr}
\hline & Total & RPD & No RPD \\
\hline Age years, mean \pm SD & $75.57 \pm 7.05$ & $76.26 \pm 6.48$ & $74.62 \pm 7.76$ \\
& & & \\
Sex, $\mathrm{n}(\%)$ & $40(45.45)$ & $21(41.20)$ & $19(51.40)$ \\
$\quad$ Male & $48(54.54)$ & $30(58.80)$ & $18(41.60)$ \\
$\quad$ Female & $65.65 \pm 20.90$ & $67.78 \pm 20.18$ & $62.48 \pm 20.48$ \\
$\begin{array}{l}\text { Follow-up months, } \\
\text { mean } \pm \text { SD }\end{array}$ & $0.13 \pm 0.14$ & $0.13 \pm 0.12$ & $0.13 \pm 0.16$ \\
$\begin{array}{l}\text { Baseline BCVA } \\
\text { logMAR, mean } \pm \text { SD }\end{array}$ & & & \\
\hline $\begin{array}{l}\text { Abbreviations: BCVA, best corrected visual acuity; } \\
\text { pseudodrusen. }\end{array}$ & & &
\end{tabular}

distribution and mean age between both groups $(P=0.39$ and 0.29 , respectively). Baseline distance visual acuity was also found to be similar across patients with and without $\operatorname{RPD}(P=0.93)$.

Tables 2 and 3 display how different image modalities were used to demonstrate the presence of RPD.

The use of more image modalities, specifically including IR and OCT, allowed for a more accurate detection of RPD, with more patients having RPD detected in multiple images. In those cases where IR and OCT were not available there was a large number of patients where RPD were visible in only one method (19 out of 24). With the use of IR and OCT this number was greatly reduced to 3 out of 28 .

Looking at Table 3 we can assess how different image modalities were used to identify RPD and how capable they were in each case. The least sensitive image modality was CFP with only $29 \%$ sensitivity. The sensitivity of RF was $33 \%$, OCT was $74 \%$, FAF was $92 \%$, and that of IR was $93 \%$. Specificity on all cases was $100 \%$ although that was due to the disease definition used on our study.

The best image modalities for RPD detection were therefore IR and FAF, closely followed by OCT. The concurrence between these image modalities was remarkable. There was only one patient where RPD were detected in IR but not in FAF and there were only two cases where RPD detected in OCT were not seen in FAF and IR. CFP and RF were strikingly less useful for RPD detection, with 36 and 34 patients, respectively, presenting with RPD that went unnoticed by these image modalities.

\section{AMD phenotypes and RPD}

Typical AMD phenotypes such as intermediate and large drusen, hyperpigmentation, and hypopigmentation were present in almost all of the eyes analyzed. Only two patients had no sign of early-AMD on CFP and no RPD on any image modality and only one patient had RPD present and no other identifiable retinal sign. Detailed in

Table 2 Number of image modalities where RPD were identified

\begin{tabular}{lccr}
\hline Image modalities & \multicolumn{3}{c}{ Patients } \\
\cline { 2 - 4 } & Group 1 & Group 2 & Total \\
\hline 1 & 3 & 19 & 22 \\
2 & 2 & 1 & 3 \\
3 & 9 & 3 & 12 \\
4 & 9 & & 9 \\
5 & 5 & & 5 \\
\hline
\end{tabular}

Group 1: Patients with all 5 image modalities; Group 2: Patients with only CFP, FAF, and RF. 
Table 3 Cross-tabulation of RPD detection on different image modalities

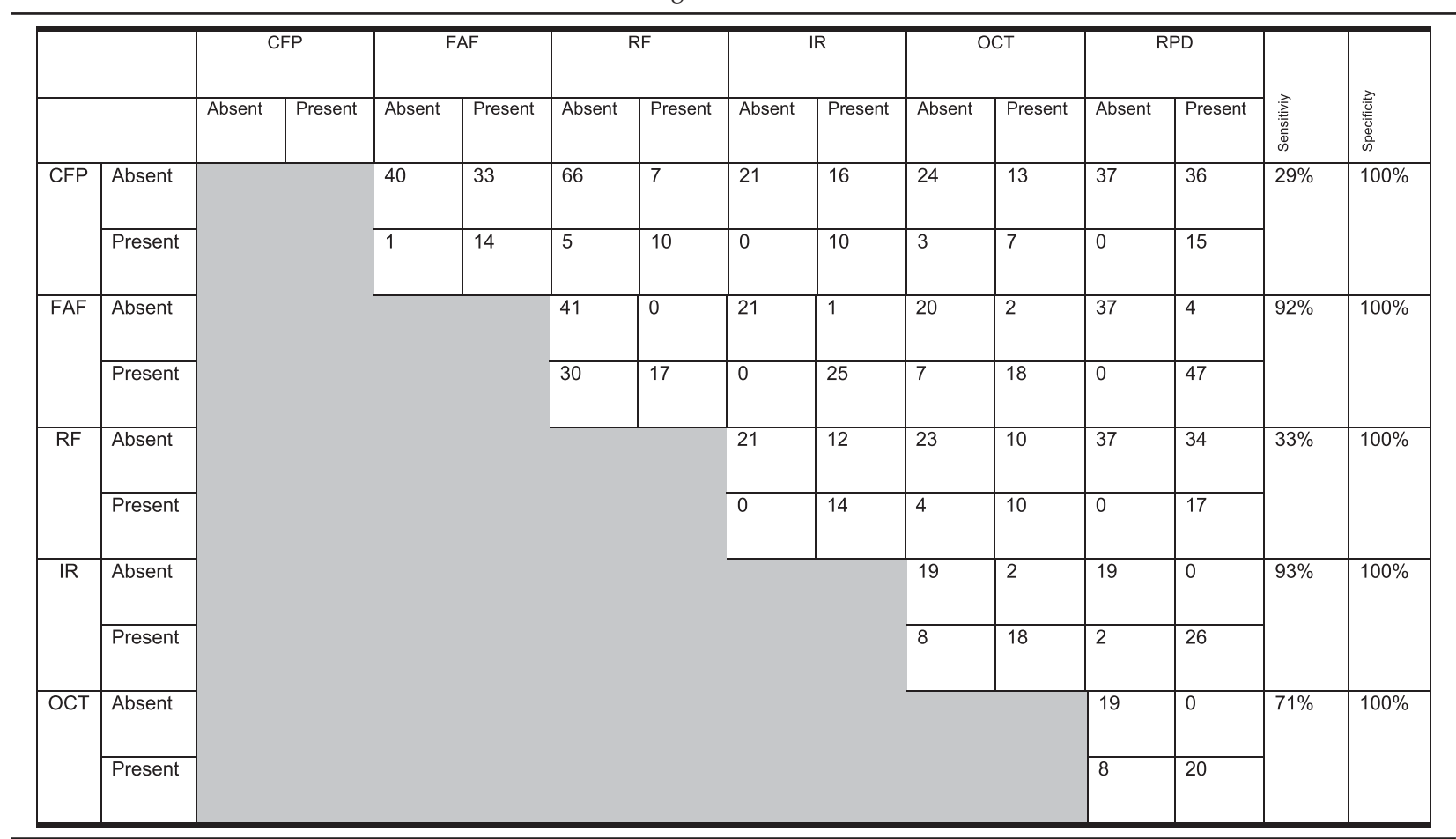

Abbreviations: CFP, color fundus photography; FAF, fundus autofluorescence; IR, infra-red; OCT, optical coherence tomography; RF, red-free; $\mathrm{RPD}$, reticular pseudodrusen.

Table 4 are the AMD features found and how they related to RPD presence. As can be seen, patients with RPD had a tendency to present other retinal signs of AMD, although such a trend could not reach statistical significance.

\section{Five-year rate of progression}

In all, $48(54.50 \%)$ of the study eyes progressed to latestage AMD, after a mean time of $30.61 \pm 20.35$ months. Of the study eyes which progressed, $81.25 \%(n=39)$ developed CNV and $18.75 \%(n=9)$ GA. RPD were present at the baseline in $33(68.75 \%)$ of the 48 eyes that progressed to late-stage AMD. Mean time from baseline until the detection of progression was not significantly different between patients with and without RPD$29.28 \pm 20.78$ and $33.64 \pm 19.76$ months, respectively $(P=0.376)$. The same was found whether progression to CNV $(26.93 \pm 20.02$ and $26.63 \pm 12.93 ; P=0.680)$ or GA $(42.00 \pm 22.27$ and $59.33 \pm 20.01 ; P=0.393)$ were considered on its own.

After correcting for age and sex, the presence of RPD was significantly associated with the development of latestage AMD (OR $=2.5, P=0.03)$. That association did not maintain statistical significance when the analysis was observed considering the development of the $\mathrm{CNV}$
Table 4 ARM features on patients with and without RPD

\begin{tabular}{|c|c|c|c|c|}
\hline & Total (\%) & $R P D(\%)$ & No RPD (\%) & P-value \\
\hline \multicolumn{5}{|c|}{ Intermediate drusen } \\
\hline Absent & 5.7 & 4.0 & 8.1 & 0.42 \\
\hline Present & 94.2 & 96.0 & 91.9 & \\
\hline \multicolumn{5}{|c|}{ Large drusen } \\
\hline Absent & 34.5 & 32.0 & 37.8 & 0.57 \\
\hline Present & 65.5 & 68.0 & 62.2 & \\
\hline \multicolumn{5}{|c|}{ Hyperpigmentation } \\
\hline Absent & 60 & 53.5 & 67.6 & 0.2 \\
\hline Present & 40 & 46.5 & 32.4 & \\
\hline \multicolumn{5}{|c|}{ Hypopigmentation } \\
\hline Absent & 60 & 51.2 & 70.3 & 0.08 \\
\hline Present & 40 & 48.8 & 29.7 & \\
\hline
\end{tabular}

Abbreviations: ARM, age-related maculopathy; RPD, reticular pseudodrusen.

subtype $(\mathrm{OR}=2.23, P=0.08)$ or $\mathrm{GA}(\mathrm{OR}=1.48, P=0.604)$ independently.

Other known markers of early-AMD were also assessed independently on their association with increased risk to progression for late-AMD. Correcting for age and sex, there was not a statistically significant association between 
progression and the presence of hypopigmentation $(\mathrm{OR}=1.93 ; P=0.18)$, hyperpigmentation $(\mathrm{OR}=1.21 ; P=0.68)$, or intermediate drusen $(\mathrm{OR}=1.78 ; P=0.54)$, when each one of them was considered on its own. Only the presence of large drusen $(\mathrm{OR}=2.93 ; P=0.025)$ was significantly linked with higher risk of progression to late-stage AMD when analyzed independently.

In a model where the presence or absence of all these known markers-hypo and hyperpigmentation, intermediate drusen, and large drusen-was considered, the association between RPD presence and increased risk of progression was still observed but no longer reached statistical significance $(\mathrm{OR}=2.35 ; P=0.089)$.

\section{Discussion}

This study analyzed the 5-year risk of progression to lateAMD on fellow eyes of patients with unilateral neovascularization and verified that the presence of RPD at baseline was a significant predictor of development of advanced disease $(\mathrm{OR}=2.5, P=0.039)$. To our knowledge, it represents the longest available follow-up on a population with neovascularization on the fellow eye and provides important information on the long-term outcomes of this particular high-risk group. These findings are in accordance with those previously observed with shorter follow-ups in similar populations. 8,9 Although important in establishing the link between RPD and increased risk of progression, the limited follow-up presented in those studies may curtail its implications in clinical practice. The extended followup considered in our study allows us to provide further new insights into the relevance of RPD for long-term management. Also, many grading schemes for AMD progression were built based on progression rates at 5 years, which emphasizes the relevance of defining this time point as an eligibility criterion. Our findings are even more remarkable if we consider that all the patients we evaluated had neovascularization on the non-study eye and, therefore, were at high risk of progression. ${ }^{16}$ Still, even in such a high-risk cohort, observing the presence of RPD rendered an additional risk, thus strengthening the importance of RPD as a long-term risk factor. Given the importance of establishing risk stratification profiles for AMD patients, the relative contribution of RPD should always be taken into account by the attending physicians when evaluating and discussing AMD patients' prognosis. The association found was marginally no longer significant when all other risk markers were considered in the statistical model. However, as was seen on our cohort, there is an important overlap between RPD and all other retinal markers. Only one patient could be found that had RPD alone, making it difficult to discern the relative contribution of RPD in relation with the remaining known phenotypes.

Despite neither one of them reaching statistical significance, there seemed to be a stronger association between RPD and CNV rather than GA development. This is controversial as several studies published report the opposite. ${ }^{8-10}$ To our knowledge, only two other studies have observed the same pattern of progression. ${ }^{17,18}$ Overall, the percentage of patients progressing to GA in our cohort $(10.22 \%)$ was relatively similar to that seen in other similar populations: $13.6 \%$ in the study by Pumariega et al, ${ }^{19} 14 \%$ in the study by Finger et $a l^{8}$ or $4.76 \%$ in the study by Hogg et al. ${ }^{9}$ We can hypothesize that our unprecedently long follow-up might have influenced this outcome. Also, fellow eyes of patients with unilateral CNV are known to be more prone to progression for late neovascular AMD when compared with the general AMD population. ${ }^{20}$ In a moderately sized sample as our own, that could also help explain why the development of GA-in smaller numbers-was not sufficient to be significantly linked with RPD.

Traditionally, RPD were identified through blue-light photography. However, the advances in retinal imaging have expanded our comprehension of retinal phenotypes and several studies have showed that the use of multiple image modalities increases our ability to identify RPD (Figure 1). 5,7-9,13,21,22 Our study incorporates this multimodal imaging approach and included 5 image methods. Similarly to other studies, $, 9,13,23$ in our cohort, IR imaging showed a superior sensitivity to identify pseudodrusen, followed by FAF. The combined use of several image modalities makes us confident that we reliably detected RPD presence in our population. In two population-based studies, the overall prevalence of RPD was $0.7 \%^{24}$ and $1.95 \%{ }^{10}$ for the general population. In AMD patients, the prevalence of RPD is notoriously higher. A higher prevalence (58\%) was found in our cohort compared with other studies $9,19,21$ that evaluated fellow eyes of patients with unilateral AMD. However, that value was very similar (58\%) to a recent report by Finger et $a l^{8}$ on the same high-risk group, using a similar multimodal approach.

The introduction of a software-assisted grading protocol allowed us to delineate more thoroughly the RPD features in different image modalities. These data might provide important information in order to better identify specific high-risk phenotypical profiles and also contribute to elucidate the mechanisms behind the formation of these lesions. This opens a potential area of research to uncover the mechanism driving the formation of RPD and progression to late-stage AMD. Integration of specific reticular patterns with anatomical findings from the RPE, choriocapillaris or choroid might help us to identify the underlying disorder. 

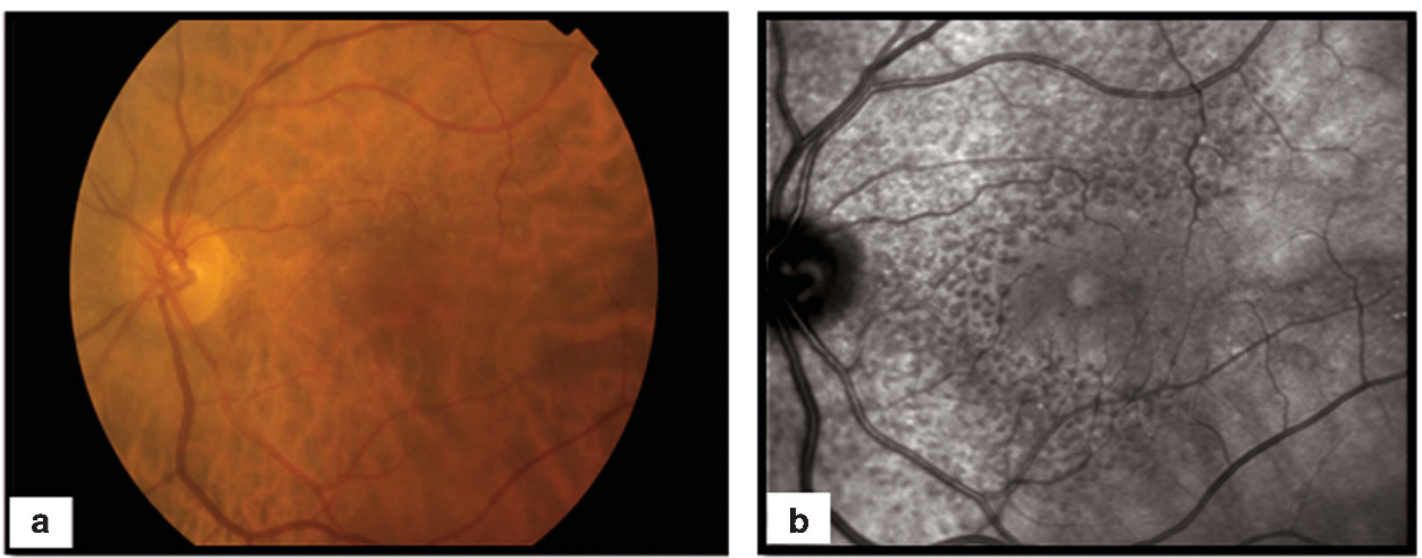

Figure 1 Example of a patient where the CFP (a) shows no remarkable features other than what appears to be some soft drusen. However, IR imaging (b) shows extensive reticular pseudodrusen distributed around the fovea.

Limitations of this study include its moderate sample size. It also represents a limited subset of high-risk AMD patients, since all participants had neovascular AMD in one eye. Among its strengths are the inclusion of a multicenter international cohort of patients, and the comprehensive retinal imaging investigation performed, assessed with a strict grading protocol. The follow-up observed in our study is also significantly longer compared to previous reports.

In conclusion, our study confirms that RPD is an independent risk factor for the 5-year progression rate in fellow eyes of patients with unilateral CNV. The long follow-up considered expands our previous knowledge on this particular AMD phenotype. Comprehensive multimodal retinal imaging and careful assessment of these high-risk lesions is recommended for all at-risk patients.

\section{Summary}

\section{What was known before}

- Reported prevalence of reticular pseudodrusen in AMD patients is highly inconsistent.

- Reticular pseudodrusen have been considered a risk sign for late-AMD but limited follow-up is available.

\section{What this study adds}

- We used a multimodal imaging approach under a strict grading protocol to reveal a high prevalence of RPD lesions in the fellow eyes of patients with unilateral AMD.

- The 5-year follow-up observed in our study is, to our knowledge, the longest reported and significantly expands our knowledge on the evolution of this retinal phenotype. The presence of RPD is associated with increased longterm risk of progression for late-stage AMD.

\section{Conflict of interest}

The authors declare no conflict of interest.

\section{References}

1 Institute NE. Prevalence of Blindness Data. 2004. Available at https://nei.nih.gov/eyedata/pvd_data (accessed on 27 February 2015).

2 Davis MD, Gangnon RE, Lee L-Y, Hubbard LD, Klein BE, Klein $\mathrm{R}$ et al. The Age-Related Eye Disease Study severity scale for age-related macular degeneration: AREDS Report No. 17. Arch Ophthalmol 2005; 123: 1484-1498.

3 Mimoun G, Soubrane GCG. Macular drusen. J Fr Ophthalmol 1990; 13(10): 511-530.

4 Klein R, Davis MD, Magli YL, Segal P, Klein BE, Hubbard L. The Wisconsin age-related maculopathy grading system. Ophthalmology 1991; 98: 1128-1134.

5 Zweifel Sa, Spaide RF, Curcio Ca, Malek G, Imamura Y. Reticular pseudodrusen are subretinal drusenoid deposits. Ophthalmology 2010; 117(2): 303-12.e1.

6 Klein R, Meuer SM, Knudtson MD, Iyengar SK, Klein BEK. The epidemiology of retinal reticular drusen. Am J Ophthalmol 2008; 145(2): 317-326.

7 Schmitz-Valckenberg S, Steinberg JS, Fleckenstein M, Visvalingam S, Brinkmann CK, Holz FG. Combined confocal scanning laser ophthalmoscopy and spectral-domain optical coherence tomography imaging of reticular drusen associated with age-related macular degeneration. Ophthalmology 2010; 117(6): 1169-1176.

8 Finger RP, Wu Z, Luu CD, Kearney F, Ayton LN, Lucci LM et al. Reticular pseudodrusen. Ophthalmology 2014; 121(6): 1252-1256

9 Hogg RE, Silva R, Staurenghi G, Murphy G, Santos AR, Rosina $\mathrm{C}$ et al. Clinical characteristics of reticular pseudodrusen in the fellow eye of patients with unilateral neovascular age-related macular degeneration. Ophthalmology 2014; 121(9): 1748-1755.

10 Joachim N, Mitchell P, Rochtchina E, Tan AG, Wang JJ. Incidence and progression of reticular drusen in age-related macular degeneration: findings from an older Australian cohort. Ophthalmology 2014; 121: 917-925.

11 Boddu S, Lee MD, Marsiglia M, Marmor M, Freund KB, Smith RT. Risk factors associated with reticular pseudodrusen versus large soft drusen. Am J Ophthalmol 2014; 157(5): 985-993.

12 Sohrab Ma, Smith RT, Salehi-Had H, Sadda SR, Fawzi AA. Image registration and multimodal imaging of reticular 
pseudodrusen. Invest Ophthalmol Vis Sci 2011; 52(8): 5743-5748

13 Ueda-Arakawa N, Ooto S, Nakata I, Yamashiro K, Tsujikawa A, Oishi A et al. Prevalence and genomic association of reticular pseudodrusen in age-related macular degeneration. Am J Ophthalmol 2013; 155(2): 260-269.e2.

14 Marques JP, Costa M, Melo P, Oliveira CM, Pires I, Cachulo ML et al. Ocular risk factors for exudative AMD: a novel semiautomated grading system. ISRN Ophthalmol 2013; 2013: 464218.

15 Cachulo ML, Lobo C, Figueira J, Ribeiro L, Laíns I, Vieira A et al. Prevalence of Age-Related Macular Degeneration in Portugal: the Coimbra Eye Study-Report 1. Ophthalmologica 2015; 233: 119-127.

16 Ferris FL, Wilkinson CP, Bird A, Chakravarthy U, Chew E, Csaky $\mathrm{K}$ et al. Clinical classification of age-related macular degeneration. Ophthalmology 2013; 120(4): 844-851.

17 Sarks J, Arnold J, Ho I-V, Sarks S, Killingsworth M. Evolution of reticular pseudodrusen. Br J Ophthalmol 2011; 95(7): 979-985.

18 Arnold J, Sarks S, Killingsworth M, Sarks J. Reticular pseudodrusen. A risk factor in age-related maculopathy. Retina 1995; 15: 183-191.
19 Pumariega NM, Smith RT, Sohrab Ma, Letien V, Souied EH. A prospective study of reticular macular disease. Ophthalmology 2011; 118(8): 1619-1625.

20 Group A-REDSR. Risk factors for the incidence of advanced age-related macular degeneration in the Age-Related Eye Disease Study (AREDS) AREDS Report No. 19 Age-Related. Ophthalmology 2005; 112(4): 533-539.

21 Cohen SY, Dubois L, Tadayoni R, Delahaye-Mazza C, Debibie C, Quentel G. Prevalence of reticular pseudodrusen in age-related macular degeneration with newly diagnosed choroidal neovascularisation. Br J Ophthalmol 2007; 91(3): 354-359.

22 Zweifel Sa, Imamura Y, Spaide TC, Fujiwara T, Spaide RF. Prevalence and significance of subretinal drusenoid deposits (reticular pseudodrusen) in age-related macular degeneration. Ophthalmology 2010; 117(9): 1775-1781.

23 Theodore Smith R, Sohrab Mahsa A, Busuioc Mihai, Gaetano Barile M. Reticular macular disease. Am J Ophthalmol 2009; 148(5): 733-743.

24 Klein R, Meuer SM, Knudtson MD, Iyengar SK, Eden B, Klein $\mathrm{K}$. The epidemiology of retinal reticular drusen ronald. Am J Ophthalmol 2008; 145(2): 317-326. 